\title{
Migration of objects and inferences across episodes
}

\author{
SHARON L. HANNIGAN \\ St. Lawrence University, Canton, New York \\ and \\ MARK TIPPENS REINITZ \\ University of Puget Sound, Tacoma, Washington
}

\begin{abstract}
Participants viewed episodes in the form of a series of photographs portraying ordinary routines (e.g., eating at a restaurant) and later received a recognition test. In Experiment 1, it was shown that objects (e.g., a vase of flowers, a pewter lantern) that appeared in a single episode during the study phase migrated between memories of episodes described by the same abstract schema (e.g., from Restaurant Episode A at study to Restaurant Episode B at test), and not between episodes anchored by different schemas. In Experiment 2, it was demonstrated that backward causal inferences from one study episode influenced memories of other episodes described by the same schema, and that highschema-relevant items viewed in one episode were sometimes remembered as having occurred in another episode of the same schematic type.
\end{abstract}

A long line of research has demonstrated that memories for stimuli and events are not holistic, indivisible entities. Rather, memories are constructed from separable parts that sometimes erroneously combine to create false memories. These parts may be encoded directly from stimuli or events, may be acquired in widely separated episodes, or may take the form of entries in schemas or scripts. For example, participants often incorrectly claim to have previously experienced new stimuli that are constructed of parts of separately experienced stimuli (see, e.g., Reinitz, Lammers, \& Cochran, 1992), mistakenly remember buildings and road signs that had been present in one environment as having occurred in a different environment (see, e.g., Albert, Reinitz, Beusmans, \& Gopal, 1999), and falsely remember having witnessed events that had not been presented but that are highly typical of the type of episode that they viewed (see, e.g., Bower, Black, \& Turner, 1979; Hannigan \& Reinitz, 2001).

Various sorts of cross-episode migrations, in which information acquired in one setting is remembered as having occurred in a different episode, have been reported in the literature. For instance, information presented on a postevent questionnaire may later be remembered as having occurred during the corresponding event (see, e.g., E. F. Loftus, Miller, \& Burns, 1978), and an individual viewed on the day that a crime is witnessed may later be remembered as having been present at the crime (Ross, Ceci, Dunning, \& Toglia, 1994). The present research addressed

Correspondence concerning this article can be addressed to S. L. Hannigan, Department of Psychology, St. Lawrence University, Canton, NY 13617 (e-mail: shannigan@ stlawu.edu). memory errors in which information acquired during one episode is later remembered as having occurred in a different episode. Our two purposes were to test for semantic constraints on these between-episodes conjunction errors and to test the types of information that may migrate from one episode to another in memory. We focused on two specific types of memory errors that we have studied extensively in recent research: memory conjunction errors and causal inference errors.

Memory conjunction errors occur when participants report a memory that is a composite of two separate experiences (see, e.g., Reinitz \& Hannigan, 2001; Reinitz et al., 1992). These errors sometimes involve the miscombination of parts of separately experienced stimuli. For instance, Jones, Jacoby, and Gellis (2001) presented participants with two sets of compound words. One set was presented visually, and the other was presented auditorily. On a subsequent recognition test, the participants tended to make incorrect positive recognition responses to new compound words (e.g., STARFISH) comprised of previously presented components (e.g., STARGAZE, CATFISH) even when those components had been studied in different modalities. Memory conjunction errors may also entail misremembering that a stimulus had been present in one episode when it had actually been present in a separate episode. Albert et al. (1999) demonstrated object-episode conjunction errors, in which landmarks (e.g., road signs, buildings) that had originally appeared in tours of different virtual environments were often remembered as having occurred in the same environment.

The source-monitoring framework proposed by Johnson and colleagues (Johnson, 1991; Johnson, Hashtroudi, \& Lindsay, 1993) predicts the sort of cross-episode mi- 
grations demonstrated by Albert et al. (1999). According to this framework, participants determine the source of a memory by applying a set of cognitive processes that are sensitive to characteristics of that memory. For instance, visual features associated with a memory are generally diagnostic that the source of the information was external rather than internal. To the extent that memory characteristics are not very diagnostic of the particular context in which an item has occurred, one would expect sourcemonitoring errors, in which the item is incorrectly assigned to an episode in which it had not originally occurred. Many studies have demonstrated that characteristics of memories change over time; as such changes occur, they may influence the accuracy of source monitoring. For instance, there is much evidence that specific episodic information rapidly becomes inaccessible following an episode. As this occurs, memory is "filled in" with generic semantic information proposed to be contained in schemas, frames, or scripts (see, e.g., Bartlett, 1932; Bower et al., 1979; Hannigan \& Reinitz, 2001; Shank \& Abelson, 1977). For example, participants may rapidly forget the specific wording of a text but remember the main ideas contained in it (see, e.g., Bransford \& Franks, 1971), or they may forget specific claims made in an advertisement (e.g., that a car has a 454-hp engine and goes from 0 to $60 \mathrm{mph}$ in $6.1 \mathrm{sec}$ ) but remember an abstraction based on those claims (e.g., that the car is powerful; Chattopadhyay \& Alba, 1988). As episode-specific information is lost, it should be increasingly hard for participants to discriminate the particular episode in which an object occurred. In accordance with this explanation, Hannigan and Reinitz (2000) demonstrated that memory conjunction errors rapidly increased in frequency as the retention interval increased.

To the extent that aspects of a given episode are abstracted into a generic type, that type should provide information that is useful for discriminating the source of an object. That is, episodic information that is diagnostic for remembering that a vase was present in Restaurant A may be rapidly forgotten and replaced with generic information that is diagnostic for remembering that the vase was present in a restaurant. This explanation predicts that there should be semantic constraints on the memorial migration of items between episodes. Specifically, such migrations should occur more frequently between episodes anchored by the same underlying schema (e.g., going to a restaurant or going grocery shopping) than between episodes anchored by different underlying schemas. The main goal of Experiment 1 was to test this prediction.

We have proposed that schematic similarity should give rise to source-monitoring errors and that such errors should increase with increasing retention interval as episodic information becomes less available. In support of these proposals, a variety of findings indicate that schematic information is used in source monitoring, and that reliance on schematic information increases as other types of information become less available. For instance, Spaniol and Bayen (2002) showed that participants tended to mis- attribute statements to a more likely source (e.g., they attributed "cold front" to a weatherman when the source had actually been a sportscaster) and that these errors were most frequently made by participants with poor item memory (e.g., those who had the least amount of episodic information available). Henkel and Franklin (1998) showed that participants were more likely to falsely remember that an imagined item (e.g., a pair of pants) had been perceived when they had perceived an item from the same semantic category (e.g., a shirt) than when they had not. This indicates that memory decisions about an object can be influenced by similar semantic information acquired from other objects. Both of these findings indicate that semantic consistency should increase source-monitoring errors relative to a semantically inconsistent condition and bolster our predictions regarding same-schema versus differentschema conjunction errors.

Causal inference errors occur when people misremember their causal inferences as external events. In a recent study by Hannigan and Reinitz (2001), participants viewed slide sequences (called action sequences) depicting typical episodes (e.g., going to a supermarket, going to a restaurant) during the study phase of a recognition experiment. Each action sequence included a causal staging slide intended to induce the participants to make an inference; for instance, in the supermarket sequence, some participants saw a slide of a woman picking up oranges from the supermarket floor. In the subsequent recognition test, the participants had a strong tendency to falsely remember a corresponding "cause" scene (e.g., the woman pulling an orange from the bottom of the stack). The sourcemonitoring framework is again useful in accounting for this result. Hannigan and Reinitz (2001) argued that the participants remembered the results of their inferences but failed to remember that the information had been internally generated. Instead, they interpreted the information as arising from externally witnessed events (a type of sourcemonitoring failure referred to as reality-monitoring errors by Johnson, 1991).

The research discussed above shows that items experienced during one episode may be misremembered as having occurred within a separate episode. Given this, it is interesting to ask whether causal inferences made during one action sequence may later be misremembered as having occurred in a different action sequence, thereby producing false recollections of that episode. For instance, if Actor 1 in Restaurant A is seen cleaning up water from his table, will participants later falsely remember having seen Actor 2, in Restaurant B, overturning his glass? Such a finding would imply that inferred events constitute items in memory that are free to migrate across episodes and would indicate that an event derived via inference constitutes a component of memory that is hierarchically equivalent to other items, such as previously seen buildings or road signs, that have been shown to migrate across episodes. Experiment 2 tested for cross-episode migration of inferred events. 


\section{EXPERIMENT 1}

Experiment 1 tested for semantic constraints on the memorial migration of objects from one episode to another. Participants viewed action sequences depicting typical episodes. Two action sequences were of trips to restaurants and involved different actors going to visually dissimilar restaurants. Two of the action sequences were "getting-up-in-the-morning"routines and showed two different actors in different homes getting out of bed, brushing their teeth, getting dressed, eating breakfast, and so on. Some of the participants saw slides containing critical objects (e.g., a vase of flowers). The subjects later received a recognition test containing new slides in which some critical objects that had occurred in one action sequence had been moved to slides from other action sequences that were either thematically similar to or thematically dissimilar from the action sequence that had originally contained the objects. For instance, a large vase viewed in Restaurant Sequence A might be present in a Restaurant Sequence B test slide (within-schema conjunction condition) or in a test slide from a morning routine sequence (betweenschemas conjunction condition). For reasons described earlier, we predicted that the participants would make more false alarms to thematically similar conjunction items than to thematically dissimilar conjunction items.

\section{Method}

Participants. Forty-eight Boston University undergraduates participated for credit in their introductory psychology classes. They were tested in 16 groups of 3 participants each. All had normal or corrected-to-normal vision.

Stimuli and Apparatus. The study and test stimuli consisted of 52 slides of naturalistic scenes taken with a Yashica T4 35-mm camera. Each scene belonged to one of six different event sequences, or episodes. Two of the episodes depicted a person going grocery shopping. These were used as primacy and recency items during the study phase, and no pictures from these episodes were presented during the test. Of the experimental episodes, two depicted a person eating at a restaurant, and two depicted a person getting up in the morning. Despite thematic redundancy, experimental episodes of the same type (i.e., episodes that were anchored to the same schema) were photographed in quite different settings using different actors in order to make them as unique as possible. For instance, the two restaurant episodes were filmed in visually dissimilar restaurants. The actors were chosen to look different in the two cases (e.g., a man with long hair in a ponytail in one case and a man with short hair in the other), and they wore very different clothing.

Four critical objects appeared in selected study and test stimuli: a red napkin, a vase of flowers, a pewter lantern, and a mug of coffee topped with whipped cream. Slides containing a given critical object were as similar as possible between episodes; for instance, the vase always appeared while someone was eating, the coffee with whipped cream always appeared when the person was cupping the coffee in his or her hands, and so on. All of the scenes were presented using Kodak slide projectors equipped with Gerbrands tachistoscopic shutters. The scenes subtended about $5^{\circ}$ of visual angle vertically and about $5^{\circ}$ horizontally. The projectors and shutters were controlled by an IBM-AT-compatible computer; timing was controlled by a clock card in the computer.

Design and Procedure. The subjects were seated before a white screen in a dimly lit room. For the study phase of the experiment, they viewed six episodes: four experimental and two fillers. The theme of two of the experimental episodes was eating at a restaurant, and the theme for the remaining two was getting up in the morning. The theme of both filler episodes was going grocery shopping. The two filler episodes were presented to control for primacy and recency effects; one was the first of the six episodes shown at study, and the other was the last, or sixth, shown. The order of presentation of the four experimental episodes alternated between restaurant and morning episode themes. Each of the six study episodes consisted of seven scenes arranged in logical sequence, with each scene appearing on the screen for $4 \mathrm{sec}$ and separated from the next by a fixed interstimulus interval of $3.5 \mathrm{sec}$. A 12-sec blank interval separated the six episodes. Before viewing the episodes, the participants were instructed to study each and every scene as well as they possibly could. They were also informed of the number of episodes they would see and were given a rough estimate of how many scenes they could expect to view per episode.

The study phase was followed by a 20 -min retention interval, during which a filler multiple-choice vocabulary test was administered. The subjects were instructed to complete as much of the test as possible in the allotted time.

The subjects were then given a recognition test that entailed viewing a series of 12 scenes and deciding for each one whether or not they had seen it during the study phase. The test utilized a 5-point confidence rating scale labeled as follows: $5=$ sure old $; 4=$ probably old $; 3=$ don't know; 2 = probably new ; and $1=$ sure new. Four of the test scenes were "old" (i.e., they had been previously presented); they were drawn from each of the four experimental study episodes. The remaining scenes were new and consisted of two conjunction foils, two control foils, and four previously unseen filler items. Conjunction foils were formed by transferring a critical object that appeared in a single scene within one episode shown at study to another scene belonging to a different episode at test. Importantly, there were two different kinds of conjunction foils: withinschema and between-schemas. Within-schema stimuli were formed when the critical object present in one restaurant episode at study was transferred to a scene from the other restaurant episode at test, or from one morning episode at study to the other morning episode at test. Between-schemas stimuli resulted when the critical object present in one of the restaurant episodes at study appeared in a scene from one of the morning episodes at test, and vice versa. Each participant received one of each type of conjunction foil. The control test stimuli were slides that contained a critical object when that object did not appear in any of the scenes viewed at study. The new filler items were slides that had not been previously presented but that involved a previously seen actor in a previously seen context. The 12 test scenes were presented for 5 sec each, in a random order. Viewing of the episodes was completely counterbalanced, with 6 participants randomly assigned to each of the eight study/test orders.

The critical objects occurred equally often in each of the study episodes across participants. To control for similarity across episodes, an individual critical object always appeared in scenes depicting the same action. For example, in all action sequences, the vase appeared in scenes in which the person was eating, the napkin in scenes in which the person was reading, and so on.

\section{Results and Discussion}

Since there was only a single test trial in each of the conjunction conditions, statistics were run using groups rather than participants as the unit of analysis. The mean confidence ratings for the various test conditions are presented in Figure 1, along with their 95\% within-participants confidence intervals (G. R. Loftus \& Masson, 1994).

An analysis of variance (ANOVA) revealed that there was a significant effect of test condition $[F(5,75)=29.56$, $\left.M S_{\mathrm{e}}=0.50, p<.001\right]$. Critical objects tended to migrate 


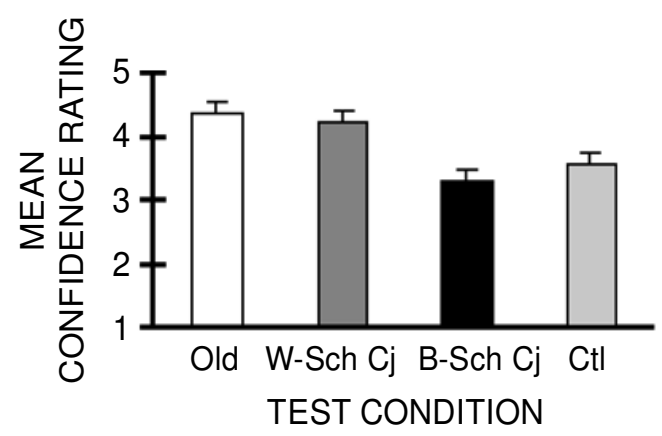

Figure 1. Results of Experiment 1: Mean confidence rating in each of the four test conditions. The error bars show the $95 \%$ within-participants confidence intervals. Old $=$ old test condition, W-Sch $\mathbf{C j}$ = within-schema conjunction condition, B-Sch $\mathrm{Cj}=$ between - schem as conjunction condition, and $\mathrm{Ctl}=$ control condition.

in memory between episodes that were thematically similar: Confidence ratings for within-schema conjunction stimuli were significantly higher than those for control stimuli (i.e., the identical stimuli when the critical item had not been presented during the study phase) $[t(15)=$ $2.89, p<.05]$. However, critical items did not tend to migrate across episode types. The comparison of betweenschemas conjunction stimuli with control pictures did not approach significance $[t(15)=-0.66]$. In short, critical objects were often incorrectly remembered as having occurred in episodes that had the same underlying thematic representation as the source sequence, but tended not to be misremembered as having occurred in episodes described by different schemas.

This finding indicates that semantic constraints exist on the migration of objects in memory from one episode to another. The present findings are consistent with the proposal that context may be defined by specific episodic information that has been encoded during an event, as well as more generic, semantic information that has been abstracted from the event. Episodic contextual information helps to avoid within-schema conjunction errors by distinguishing the specific episode in which an item occurred, and abstracted generic information helps to avoid between-schemas conjunction errors by specifying the event type in which an item occurred.

\section{EXPERIMENT 2}

Recently, Hannigan and Reinitz (2001) showed that backward causal inferences sometimes cause participants to mistakenly "remember" scenes representative of those inferences. In that study, participants viewed episodes (e.g., going grocery shopping, attending a lecture) in which either a cause slide or an effect slide was presented. Forward-inference errors occurred when participants who viewed a cause slide (e.g., taking an orange from the bottom of the stack) during the study session later confidently recognized its corresponding effect slide (e.g., picking up fallen oranges) when the effect slide had not appeared at study. Backward-inference errors occurred when only the effect slide (e.g., picking up fallen oranges) was shown at study and participants later falsely claimed to have seen a slide representing a probable cause of that effect (e.g., taking an orange from the bottom of the stack). The retention interval was $20 \mathrm{~min}, 1$ day, or 2 days. The main findings were that (1) participants were more likely to falsely recognize cause slides when they had viewed the corresponding effect slide than when they had not viewed the effect slide; (2) there was no forward-inference effect-that is, viewing a cause slide did not increase the likelihood of falsely recognizing the effect; and (3) the backward-causalinference effect increased with increasing retention interval.

The main objective of Experiment 2 was to test whether causal inferences, like objects, represent units in memory that can migrate across episodes anchored by the same abstract schema. As a result, the structure of Experiment 2 differed from that of Experiment 1 in an important way. Whereas Experiment 1 tested for conjunction errors both within and between episode types (i.e., same schema vs. different schemas), all of the conjunctions that were tested in Experiment 2 were between schematically similar episodes. As did Hannigan and Reinitz (2001), we tested whether people falsely recognize cause scenes implied by previously presented effect scenes (backward-inference, same-episode condition). However, we also included a condition in which, after showing an effect slide in one episode at study, we showed a corresponding cause slide involving the central characters and environment of a different, schematically similar episode at test (backwardinference conjunction condition). For instance, if an effect slide depicting Sarah picking oranges up off the floor of Jimmy's Market was shown as part of Grocery Episode A at study, then a cause slide omitted from the other grocery shopping episode (Grocery Episode B) presented at study - that is, a slide of Astrid grabbing an orange from the bottom of the stack at Market Supreme-was shown at test. If inferences migrate across episodes in the same way that objects do, this would imply that objects and inferences may both serve as building blocks from which memories of episodes are constructed. If, on the other hand, inferential errors occur only within a given episode, this would imply that inferences are strongly bound to a specific episodic context, or that they modify the underlying episodic representation for a specific episode.

In addition to testing causal inference errors, Hannigan and Reinitz (2001) tested the memorial consequences of schema-typical items. Participants viewed high-schemarelevant test slides depicting highly schema-typical events (e.g., ordering food in a restaurant action sequence) and low-schema-relevant test slides depicting schema-consistent but not highly schema-typical events (e.g., sipping water in a restaurant). For both old and new test items, the participants were more confident that they had previously seen high-schema-typical than low-schema-typical test items. Another objective of Experiment 2 was to test whether high-schema-relevant and low-schema-relevant 
scenes migrate across episodes anchored by the same schema. For example, if a high- (or low-) schema-relevant scene appeared in one restaurant episode at study (e.g., Pearl taking an order in Kelly's Diner), then a thematically similar scene that had been omitted from the other restaurant episode presented at study was shown at test (e.g., Flo taking an order in Mel's Kitchen). The decision to include high- and low-schema-relevant conjunction conditions is based on the idea that the more typical an item is, the more freely it should migrate to other episodes anchored by the same abstraction. This is expected because, in theory, high-schema-relevantscenes engage schema entries whose activation levels were boosted at study by thematic analogues. The residual activation of schema entries by study stimuli, together with their recent activation by test stimuli, presumably invokes a powerful schematic context that gives rise to strong feelings of familiarity. Moreover, since schema entries are by definition generic, they do not contain episode-specific features that would be useful in discriminating the specific episode in which they had occurred. In contrast, low-schema-relevant items, which are not abstractly represented in schematic memory, do not activate specific schema entries. Therefore, their context is likely the unique episodes in which they had occurred. It is predicted that participants will reject low-schema-relevant conjunction foils as old because these items can be discriminated from thematically similar low-schema-relevant study stimuli on the basis of their different episodic contexts.

Finally, as did Hannigan and Reinitz (2001), we tested for high- and low-schema-relevant gap-filling errors. Such errors occur when participants falsely recognize slides depicting high- or low-schema-relevant events not viewed during any of the study sequences. In previous research, participants had a tendency to falsely recognize highschema-relevant but not low-schema-relevant slides. Aside from being of interest in their own right, the two schemarelevant gap-filling conditions served as controls for the high- and low-schema-relevant conjunction conditions.

The participants in Experiment 2 studied eight episodes featuring four types of routines: going grocery shopping, eating at a restaurant, getting up in the morning, and attending a lecture. Critically, for each routine type there were two distinct episodes - that is, episodes that were thematically similar to but situationally dissimilar from each other (e.g., Restaurant Episode A/Restaurant Episode B, Lecture A/Lecture B). Following a 15-min, 1-day, or 3- day interval, the participants received a recognition test that involved rating their confidence that they had seen individual scenes during the study phase. In order to test for within-episode and between-episodes causal inference errors, the test included backward-inference, same-episode items (new slides depicting the cause of an effect that had been previously viewed in that action sequence) and backward-inference conjunctionitems (new slides depicting a cause of an effect that had been viewed in a different action sequence of the same generic type), as well as backward-inference control items (cause slides whose corresponding effect slide had not been presented in any action sequence). To test the effects of schema relevance, the test also included old high-schema-relevantitems, old low-schema-relevant items, new high-schema-relevant items, new low-schema-relevant items, high-schemarelevant conjunctionitems (in which a high-schema-relevant event that had occurred in one episode was depicted as having occurred in another episode), and low-schemarelevant conjunction items. If backward inferences migrate across episodes, then there should be more false alarms to backward-inference conjunctionitems than to their corresponding backward-inference control items. If highly typical events tend to migrate across episodes, then there should be more false alarms to high-schema-relevant conjunction items than to new high-schema-relevant items.

\section{Method}

Participants. One hundred forty-four Boston University introductory psychology students participated in this experiment for course credit. Forty-eight participants were randomly assigned to each of three retention interval conditions (15 min, 1 day, and 3 days). All the participants were tested in groups of 3 and had normal or corrected-to-normal vision. None had participated in the previous experiment.

Stimuli and Apparatus. The study and test stimuli consisted of 112 color photographic slides of naturalistic scenes. Each scene belonged to one of eight different episodes: Restaurant Episodes A and B, Lecture Episodes A and B, Morning Routine Episodes A and B, and Grocery Shopping Episodes A and B. Each episode consisted of 14 scenes: 4 causal, 4 schema-based (i.e., 2 high- and 2 low-schemarelevant), and 6 nonexperimental. As in Experiment 1, the sameschema episodes were intentionally as distinct as possible, involving different actors in visually dissimilar settings. Test slides were assigned to the high- and low-schema-relevant conditions on the basis of norms established by Bower et al. (1979; for details, see Hannigan \& Reinitz, 2001, pp. 933-934). There were two causal sets per episode, each consisting of one cause slide and one effect slide, as is shown in Table 1. The apparatus used in Experiment 1 was also used here.

Table 1

Causal Sets of Stimuli Presented in Experiment 2

\begin{tabular}{|c|c|c|}
\hline Episode & Cause & Effect \\
\hline Restaurant & $\begin{array}{l}\text { accidentally knocking over a glass of water } \\
\text { finding a hair in the soup }\end{array}$ & $\begin{array}{l}\text { mopping up spilled water } \\
\text { complaining to the waitress }\end{array}$ \\
\hline Lecture & $\begin{array}{l}\text { tilting back in desk } \\
\text { called on by professor }\end{array}$ & $\begin{array}{l}\text { toppling over } \\
\text { "I don't know the answer" gesture }\end{array}$ \\
\hline Grocery shopping & $\begin{array}{l}\text { taking an orange from the bottom of the orange stack } \\
\text { grocery bag breaking through }\end{array}$ & $\begin{array}{l}\text { picking oranges up off the floor } \\
\text { gathering groceries off the sidewalk }\end{array}$ \\
\hline Morning routine & $\begin{array}{l}\text { fumbling with clock radio alarm } \\
\text { catching finger on bowl while scrambling eggs }\end{array}$ & $\begin{array}{l}\text { clock radio dangling off night table } \\
\text { cleaning up eggs }\end{array}$ \\
\hline
\end{tabular}


Table 2

Examples of Backward Causal, High- and Low-Schema-Relevant Gap-Filling, and High- and Low-Schema-Relevant Conjunction Conditions Used in Experiment 2

\begin{tabular}{|c|c|c|c|}
\hline Episode & Study Items & Test Items & New Test Conditions \\
\hline Restaurant A & Effect $1, \varnothing$ HiSR, Ø LoSR & HiSR, LoCj & High-schema-relevant, low-schema-relevant conjunction \\
\hline Lecture A & LoSR, Ø Effect $1, \varnothing$ Effect $2, \varnothing$ HiSR & Cause 2 & Backward-inference-betwe \\
\hline Grocery Shopping A & Effect $2, \varnothing$ Effect $1, \varnothing$ LoSR, $\varnothing$ HiSR & $\begin{array}{l}\text { Cause } 2 \text {, Cause } 1 \\
\text { LoSR, HiCj }\end{array}$ & $\begin{array}{l}\text { Backward-inference-within, backward-inference control } \\
\text { Low-schema-relevant, high-schema-relevant conjunction }\end{array}$ \\
\hline Morning Routine A & HiSR, Ø Effect 2, Ø LoSR & & \\
\hline Restaurant B & LoSR, Ø Effect 1, Ø Effect 2, Ø HiSR & Cause 1, Cause 2 & Backward-inference-between, backward-inference control \\
\hline Lecture B & Effect $1, \varnothing$ HiSR, $\varnothing$ LoSR & HiSR, LoCj & High-schema-relevant, low-schema-relevant conjunction \\
\hline Grocery Shopping B & HiSR, Ø Effect 2, Ø LoSR & & \\
\hline Morning Routine B & Effect 2, Ø Effect 1, Ø LoSR, Ø HiSR & $\begin{array}{l}\text { Cause } 2 \text {, Cause } 1 \\
\text { LoSR, HiCj }\end{array}$ & $\begin{array}{l}\text { Backward-inference-within, backward-inference control } \\
\text { Low-schema-relevant, high-schema-relevant conjunction }\end{array}$ \\
\hline
\end{tabular}

Note-Cause 1 = cause slide for Causal Set 1; Effect 1 = effect slide for Causal Set 1; Cause 2 = cause slide for Causal Set 2; Effect 2 = effect slide for Causal Set 2; HiSR = high-schema-relevant slide; LoSR = low-schema-relevant slide; HiCj = high-schema-relevant conjunction slide; LoCj $=$ low-schema-relevant conjunction slide; $\varnothing=$ stimuli not shown at study.

Design and Procedure. For the study phase, the participants viewed eight episodes, each consisting of nine scenes arranged in logical order. Four filler slides - two shown at the beginning and two at the end of the study session-controlled for primacy and recency effects. The episodes were presented in different study orders, with two constraints: that one of each episode type was among the first four episodes shown and the other among the last four shown, and that no two episodes of the same type were shown in succession. Each action sequence was constructed to contain two effect scenes, which set the stage for backward-inference same-episode and backwardinference conjunction errors, and two schema-based scenes, which set the stage for the high- and low-schema-relevant conjunction conditions. During a given study session, nine slides were presented in each of the eight study sequences. To create the necessary test conditions, some slides were not presented during the study phase; for instance, some effect slides were not shown during study in order to create the backward-inference control condition, and some high- and low-schema-relevant slides were not shown at study in order to create the new high-schema-relevant, new low-schema-relevant, highschema-relevant conjunction, and low-schema-relevant conjunction conditions. Table 2 provides an example of how particular slides were withheld from the study phase in order to create the necessary new test conditions. Eight different study orders were necessary for all of the causal sets (two per episode) and schema-based stimuli to be presented an equal number of times. The study stimulus timing parameters were identical to those of Experiment 1.

Following a retention interval of $15 \mathrm{~min}, 1$ day, or 3 days, the participants received a 24 -item recognition test that utilized the same five-point confidence rating scale that was used in Experiment 1 . The test included old items, half of which were high-schema-relevant and the other half low-schema-relevant, and new items in eight different conditions. The new conditions were backward-inference same episode (two items: cause scenes implied by effect scenes shown at study from the same episode), backward-inference conjunction (two items: cause scenes implied by effect scenes shown at study from a different episode of the same schematic type), backward-inference control (two items: cause slides shown when corresponding effect slides were not presented at study), new high-schema-relevant (two items: scenes typical of an episode type that did not appear in any of the study episodes and were not thematically similar to any of the scenes shown at study), new low-schema-relevant (two items: nontypical scenes that did not appear in any of the study episodes and were not thematically similar to any of the scenes shown at study), high-schema-relevant conjunction (two items: high-schema-relevant scenes situationally different from but thematically identical to highschema-relevant scenes shown at study), and low-schema-relevant conjunction (two items: low-schema-relevant scenes situationally different from but thematically identical to low-schema-relevant scenes shown at study).

Each of the 24 test stimuli appeared on screen for $4 \mathrm{sec}$. Test order was random, with the constraint that consecutive test slides were never drawn from the same episode. Different random test orders were used for each group, with the constraint that for each 15-min group there was a 1-day and a 3-day group that received the identical study and test items in the identical order. All high- and lowschema-relevant stimuli were used equally often as old and as new test items.

\section{Results and Discussion}

Mean confidence ratings for the various test conditions across the three experimental sessions are shown in Figure 2 . The error bars reflect the $95 \%$ within-participants confidence intervals (G. R. Loftus \& Masson, 1994). ANOVAs showed significant effects of retention interval $\left[F(2,141)=23.85, M S_{\mathrm{e}}=1.50, p<.001\right]$ and test condition $\left[F(8,1128)=123.94, M S_{\mathrm{e}}=0.77, p<.001\right]$. The retention interval $\times$ test condition interaction was also significant $\left[F(16,1128)=3.44, M S_{\mathrm{e}}=0.77, p<.001\right]$.

The primary purpose of the experiment was to test whether inferences can migrate across episodes. Confidence ratings were significantly higher for backward-inference conjunction stimuli than for backward-inference control stimuli for all three retention intervals (all $p \mathrm{~s}<.05$ ). This may indicate that inferences that the participants made while viewing one episode can be misapplied to another episode described by the same abstract script. However, mean confidence ratings in the backward-inference conjunction condition were less than 3 (e.g., don't know) for all three retention intervals. The claim that inferences migrate across similar episodes is therefore not strongly supported by the confidence data; instead, the participants may simply have been less confident of their "new" responses in the between-episodes than in the control condition.

To further explore the possibility that inferences may migrate across episodes, we performed an additional analysis. Confidence responses of 4 and 5 were respectively labeled as probablyold and sure old on the response sheets. It was therefore possible to test for differences in 


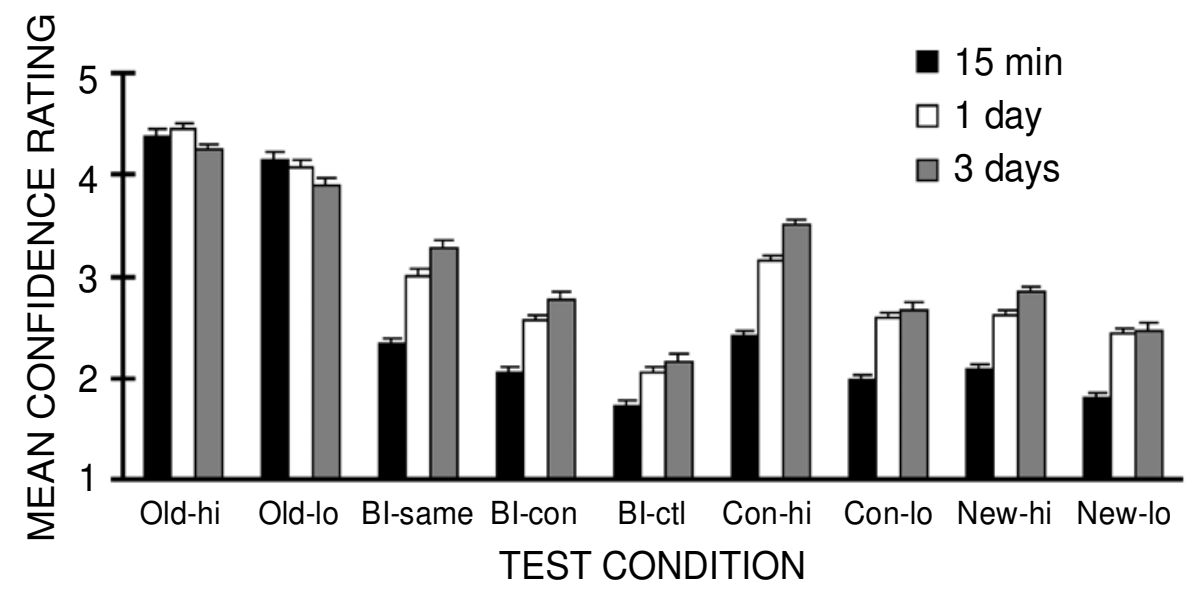

\begin{abstract}
Figure 2. Results of Experiment 2: Mean confidence rating in each of the nine test conditions across retention intervals of $15 \mathrm{~min}, 1$ day, and 3 days. The error bars show the $95 \%$ within-participants confidence intervals. Old-hi = old high-schema-relevant condition; Old-lo = old low-schema-relevant condition; BI-same = backward-inference same-episode condition; BI-con = backward-inference conjunction condition; BI-ctl = backward-inference control condition; Con-hi $=$ high-schemarelevant conjunction condition; Con-lo = low-schema-relevant conjunction condition; New-hi = new high-schema-relevant condition; New-lo = New low-schema-relevant condition.
\end{abstract}

number of "old" responses among the three backwardinference conditions by counting and analyzing the number of " 4 " and " 5 " responses made by each participant in each of the conditions. The proportion of "old" responses in the three backward-inference conditions are shown for each of the retention intervals in Figure 3. An ANOVA showed significant main effects of both test condition $\left[F(2,282)=25.60, M S_{\mathrm{e}}=0.370, p<.001\right]$ and retention interval $\left[F(2,141)=15.13, M S_{\mathrm{e}}=0.434, p<.001\right]$. The interaction was not significant $[F(4,282)=0.59]$. Separate ANOVAs were performed to compare the backward-inference same-episode and backward-inference conjunction conditions with the control condition, and in both cases the participants made significantly more "old" responses in the experimental condition than in the control condition $\left[F(1,141)=57.62, M S_{\mathrm{e}}=0.321\right.$, and $F(1,141)=$ $6.81, M S_{\mathrm{e}}=0.372$, respectively; both $\left.p \mathrm{~s}<.05\right]$. This indicates that false memories occurred in both backwardinference conditions. Most importantly, the participants were not only less confident of their "new" responses to stimuli in the backward-inference conjunction condition, but were also more likely to respond that they had previously seen those stimuli. However, it is also important to note that the participants gave significantly lower confidence ratings to backward-inference conjunction stimuli than to backward-inference same-episode stimuli $[t(143)=-3.48$, $p<.01]$ and were significantly more likely to respond that backward-inference same-episode stimuli were old $[t(143)=4.23, p<.01]$. Thus, shifts in memory based in backward causal inference are most likely to occur within the specific episode in which the inference was made.

A second finding is also consistent with the notion that memories may migrate across similar episodes. Highschema-relevant conjunction items (new slides whose ac- tions were depicted in slides from a separate episode) received significantly higher confidence ratings than did new high-schema-relevant items whose actions had not been depicted in a separate episode $[t(143)=4.92, p<.001]$. Furthermore, the mean confidence rating in the highschema-relevant conjunction condition was well above 3, indicating that the participants tended to make "old" responses to these stimuli. This tendency to misremember old high-schema-relevant items as occurring in a different episode is not due simply to visual similarity with studied scenes, since the effect is much larger for high-schema-

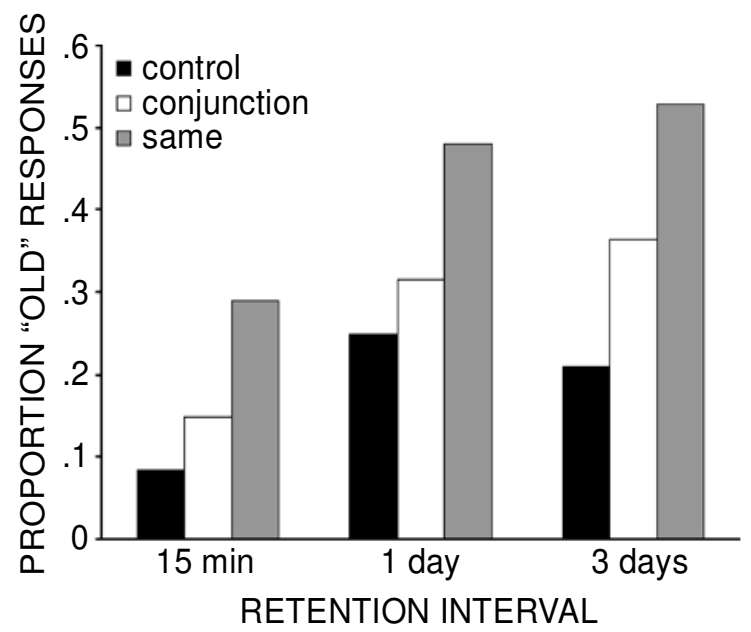

Figure 3. Results of Experiment 2: Mean proportion of "old" responses in the three backward-inference conditions for each of the three retention intervals. Same $=$ backward-inference sameepisode condition, and conjunction $=$ backward-inference conjunction condition. 
relevant than for low-schema-relevant items and since both types of items should be about equally visually similar to studied items. The pattern of results indicates that old high-schema-relevant items are more generic, and less distinctive, than are low-schema-relevant items. A possible explanation is that high-schema-relevantitems may activate preexisting generic schema entries, reducing the amount of new episodic information that is encoded from those items. As a result, less diagnostic source information may be available for these items, increasing the likelihood that participants may tend to identify them on the basis of familiarity rather than recollection (see, e.g., McDermott, 1996).

Unlike in Hannigan and Reinitz (2001), the confidence difference between high- and low-schema-relevant new conditions in both the 15-min session and the 1-day session approached but did not reach significance $(p s>.05)$. Greater confidence in new high-schema-relevant relative to new low-schema-relevant stimuli did strongly emerge, however, in the 3-day session $[t(47)=3.97, p<.001]$.

Finally, regarding effects of retention interval, the mean confidence ratings for all new test conditions significantly climbed from the 15-min delay separating study and test to the 1-day delay (all $p \mathrm{~s}<.05$ ), with no significant changes in either old condition. From the 1-day to the 3-day delay, there were no significantincreases in mean ratings for any of the test conditions, although numerically all of the changes were in the expected direction. These findings suggest that both correct and false scene recognition stabilizes within a period of 1 day after perception, and stand in contrast to the results obtained in Hannigan and Reinitz (2001), in which backward inference and new high-schemarelevant means significantly increased from a 1- to a 2-day delay. It is possible that the addition of an extra retention interval day in the present experiment allowed for greater consolidation of recent memories. Indeed, the effects of some manipulations known to interfere with eyewitness identification-for instance, the "overshadowing effects" produced when witnesses provide verbal descriptions of faces (Pezdek \& Finger, 1997)—have been shown to become less pronounced at longer retention intervals.

\section{GENERAL DISCUSSION}

There were six important findings of this study. First, it was shown that objects were semantically constrained so that they freely migrated in memory only across episodes described by the same abstract schema. Second, it was demonstrated that effect scenes viewed during one episode caused the participants to be less confident when rejecting new cause scenes from a separate but schematically similar episode. Third, it was demonstrated that backward inferences made during one episode can sometimes lead to false memories for other episodes described by the same schema. Fourth, backward-inference errors occurred significantly more frequently within an episode than across episodes. Fifth, the incidence of high-schema-relevant conjunction errors was greater than that of low-schema- relevant conjunctionitems. Sixth, mean confidence ratings for same-episode and between-episodes inference conditions and high- and low-schema-relevant source misattribution conditions significantly increased from the $15-\mathrm{min}$ delay separating study and test to the 1-day delay, with no increase observed from the 1-day to the 3-day delay.

\section{Constraints on Migration of Objects Between Episodes}

Albert et al. (1999) demonstrated that objects experienced in one episode migrated in memory so that they were later misremembered as having occurred in a different episode. In Experiment 1 of the present study, we replicated Albert et al.'s finding with different stimuli and extended it by showing that object migration occurs only between episodes anchored by the same abstract schema. The implication is that items that had occurred during an episode may be associated with abstracted, generic contextual information that is useful for remembering the type of episode in which the items had occurred, but not for remembering the specific episode in which they had occurred. This progression from specific to generic contextual information is probably characteristic of much real-world remembering. For instance, it is easy to imagine initially remembering having read a news item in a specific issue of the New York Times, and later simply remembering that the item had occurred in a newspaper.

Previous research has tended to imply that the use of schematic information in source monitoring reflects a guessing bias (e.g., Bayen, Nakamura, Dupuis, \& Yang, 2000; Spaniol \& Bayen, 2002). In those studies, information arising from a low-probability source was later attributed to a high-probability source. In those cases, participants' knowledge about sources biased their memory responses. In the present Experiment 1, critical objects were chosen to be equally probable in either schematic context and were studied equally often in both schematic contexts. The present research therefore shows that abstracted schematic information can enhance source monitoring by preventing between-schemas conjunction errors in a way that is unrelated to guessing.

\section{Migration of Inferences Across Episodes}

A major finding of Experiment 2 was that backward causal inferences influenced memory not only for the specific episode for which the inference was made, but for other, schematically similar episodes as well. The participants were less confident of their "new" responses in the backward-inference conjunction condition than of those in the backward-inference control condition. This decrease in confidence is predicted by the source-monitoring framework that motivated the research. The reason is that schematic context can provide information that may be used to attribute an item to its source. When two episodes share schematic context, the diagnostic usefulness of this information is eliminated, so that less total diagnostic information is available for source monitoring. 
There were also more "old" responses in the backwardinference conjunction condition than in the control condition, indicating that inferences were sometimes misapplied to other episodes described by the same schema, thereby giving rise to false memories for those episodes. However, the results also indicate that between-episodes inferences are considerably less vulnerable to false recognition than are inferences based on a single episode. That is, backward causal inferences, while not confined to the episodes in which they occurred, are to some degree constrained by them. An explanation of this pattern of results is that information inferred during a specific episode may be associated with either episodic or generic contextual information. Episodic information allows participants to assign that information to the specific episode in which it was inferred. To the extent that information is sometimes associated with episodic information, one would expect more within-episode than between-episodes backwardinference errors. Moreover, because episodic memory is thought to decline rapidly over time (see, e.g., Bower et al., 1979; Tulving, 1985), one would expect more crossepisode migration errors with increasing retention interval. Previous studies showing an increase in both memory conjunction errors (Hannigan \& Reinitz, 2000, 2003) and backward-inference errors (Hannigan \& Reinitz, 2001) over time are consistent with this explanation.

As was predicted, errors in the backward-inference conjunction condition increased relative to the control condition as retention interval increased. However, one prediction was not confirmed in Experiment 2. Specifically, one would assume that backward-inference errors would increase more over time in the backward-inference conjunction condition than in the backward-inference same-episode condition. The reason is that, with time, episodic information (which insulates participants from between-episodes, but not from within-episode, backwardinference errors) should be forgotten. There was no evidence of such an interaction in either the confidence data or the proportion "old" data from Experiment 2. However, it is important to note that the results reflect quite complex source-monitoring processes. For instance, even within an episode, causal-inference errors are likely to result from source-monitoring failures, in which participants remember information that they had inferred during the study phase, but mistakenly attribute the source of that information to an externally witnessed slide. Clearly, the specific processes that give rise to these errors, and the influence of the passage of time on them, remain to be fully understood.

The finding that the participants sometimes mistook backward-inference conjunction foils for scenes they had earlier viewed is consistent with compositionality, a notion central to the computational theory of mind (Pinker, 1997) - namely, that complex knowledge (e.g., propositions) consists of smaller units of knowledge that are "flexibly and systematically" related to each other (Holyoak \& Kroger, 1995; Pinker, 1997). It is possible that postulated elements of visual knowledge-for example, features (Reinitz et al., 1992), objects (Albert et al., 1999; Experiment 1 of this study), and causal inferences (Experiment 2 of this study) — are also represented as "composable units" (Holyoak \& Kroger, 1995) that can be freely manipulated in declarative memory, resulting in the construction of new knowledge (Eichenbaum, 1997; Grafman, 1995; Moscovitch, 1995). This argument refers to the function of these elements rather than to their representations; for instance, schema-based entries are generic and semantic, whereas memories for individual objects are largely episodic and visual. Despite these differences, both types of items can serve as units from which memories are composed.

\section{Schema-Relevant Conjunction Errors}

In Experiment 1, we demonstrated that objects may migrate in memory between episodes described by the same schema or script, and in Experiment 2 we showed that high-schema-relevantevents witnessed during one episode often migrate in memory so that they are later remembered as having been witnessed during a different episode described by the same schema or script. Low-schemarelevant events were less likely to migrate across episodes, presumably because they are less generic and more episode specific. The pattern of results demonstrates that generic, schematic information plays a critical role in the migration of objects and events from one episode to another.

These findings are consistent with the widely accepted proposal that script memory consists of generic representations of the typical properties of multiple, thematically similar episodes (Bower et al., 1979; Shank \& Abelson, 1977). When typical events are encountered in a given episode, they may serve as retrieval cues for the schema of that episode, with activated script entries providing relevant context for interpreting familiar situations. In Experiment 2, heightened schema activation due to the combined effects of thematically identical high-schemarelevant study stimuli and test foils may have led to errors in which the participants recognized a familiar abstract concept but failed to discriminate the episodic context in which they had originally experienced the concept. Furthermore, activation of schema entries during an event may reduce the acquisition of episodic information for those high-schema-relevant events, so that, relative to low-schema-relevant events, less information that is diagnostic of the specific episode in which those events had originally occurred is encoded into memory.

In contrast, it has been proposed that atypical eventsthat is, those presumably not represented schematicallyare first distinguished in memory (a process referred to as stimulus differentiation; Gluck \& Myers, 1995), so that they later may be bound in novel ways, free of the conditions under which they were acquired (Eichenbaum \& Bunsey, 1995). The differentiation and subsequent association of novel stimulus representations may be supported by explicit or declarative memory (Dusek \& Eichen- 
baum, 1997; Eichenbaum, 1997), which, when indexed by "remember" responses, signifies conscious recollection of an item's source (Gardiner \& Parkin, 1990; Tulving, 1985). Our findings suggest that source information present in the explicit memory traces of low-schema-relevant events was diagnostic of the specific episodes in which they occurred. That is, the participants were able to use source information to discriminate between thematically analogous low-schema-relevant study and test stimuli, leading to the correct rejection of low-schema-relevant conjunction foils.

In summary, high- and low-schema-relevant events seem to be associated with different types of contextual information. The different context types can be seen as adaptive, with schematic context providing relevant background in a familiar setting, obviating the need to encode source information, and episodic context enabling new or unique information to be freely manipulated (e.g., reasoned out, understood) in relation to the circumstances in which it was acquired (e.g., its source).

\section{Final Comments}

As is the case whenever new varieties of memory errors are discovered, the present research has applied as well as theoretical implications. Witnesses may sometimes remember a critical item as having been present at a crime scene if a similar object had been viewed in a similar setting at some other time. Causal inference errors have special relevance to eyewitness issues, as people tend to confidently remember seeing acts that they had never witnessed but only inferred. The present research shows that such errors may occur across episodes. It is therefore conceivable that inferences made while reading, or while watching a movie, might be misapplied to thematically similar events that are witnessed later.

It is possible that causal inferences, like analogies (Wharton, Holyoak, \& Lange, 1996), are represented in the brain at multiple levels (e.g., on a continuum ranging from concrete/specific to abstract/general). Wharton et al. have provided evidence that a single abstract analogy (i.e., a thematic analogue) can serve as a retrieval cue for multiple, domain-specific episodes. Further research is needed to test whether causal inferences are resistant to migrations across episode types. Finally, causal inferences represent only a single type of inference. It would be interesting to test the extent to which other types of inferences give rise to false memories, and the extent to which inferred information may migrate between episodes. The extent to which a particular type of inference is resistant to such migration should be related to the specific function of that inference type.

\section{REFERENCES}

Albert, W., Reinitz, M. T., Beusmans, J., \& Gopal, S. (1999). Role of attention in spatial learning during simulated route navigation. Environment \& Planning A, 31, 1459-1472.

BARTLETT, F. C. (1932). Remembering: A study in experimental and social psychology. Cambridge: Cambridge University Press.
Bayen, U. J., Nakamura, G. V., Dupuis, S. E., \& Yang, C. L. (2000). The use of schematic knowledge about sources in source monitoring. Memory \& Cognition, 28, 480-500.

Bower, G. H., Black, J. B., \& Turner, T. J. (1979). Scripts in memory for texts. Cognitive Psychology, 11, 177-220.

Bransford, J., \& Franks, J. J. (1971). The abstraction of linguistic ideas. Cognitive Psychology, 2, 331-350.

Chattopadhy ay, A., \& Alba, J. W. (1988). The situational importance of recall and inference in consumer decision making. Journal of Consumer Research, 15, 1-12.

Dusek, J., \& Eichenbaum,H. (1997). The hippocampus and memory for orderly stimulus relations. Proceedings of the National Academy of Sciences, 94, 7109-7114.

EICHENBAum, H. (1997). Declarative memory: Insights from cognitive neurobiology. Annual Review of Psychology, 48, 547-572.

Eichenbaum, H., \& Bunsey, M. (1995). On the binding of associations in memory: Clues from studies on the role of the hippocampal region in paired-associate learning. Current Directions in Psychological Science, 4, 19-23.

Gardiner, J. M., \& Parkin, A. J. (1990). Attention and recollective experience in recognition memory. Memory \& Cognition, 18, 579-583.

Gluck, M. A., \& Myers, C. E. (1995). Representation and association in memory: A neurocomputational view of hippocampal function. Current Directions in Psychological Science, 4, 23-29.

Grafman, J. (1995). Similarities and distinctions among current models of prefrontal cortical functions. In J. Grafman, K. J. Holyoak, \& F. Boller (Eds.), Structure and functions of the human prefrontal cortex (Annals of the New York Academy of Sciences, Vol. 769, pp. 337368). New York: New York Academy of Sciences.

Hannigan, S. L., \& Reinitz, M. T. (2000). Practical factors that influence the likelihood of false recognition: Influences of temporal factors on memory conjunction errors. Applied Cognitive Psychology, 14, 309-321.

Hannigan, S. L., \& Reinitz, M. T. (2001). A demonstration and comparison of two types of inference-based memory errors. Journal of Experimental Psychology: Learning, Memory, \& Cognition, 27, 931-940.

Hannigan, S. L., \& Reinitz, M. T. (2003). Effects of divided attention and the passage of time on memory conjunction errors. Manuscript in preparation.

Henkel, L. A., \& Franklin, N. (1998). Reality monitoring of physically similar and conceptually related objects. Memory \& Cognition, 26, 659-673

HolyoAK, K. J., \& Kroger, J. K. (1995). Forms of reasoning: Insight into prefrontal functions? In J. Grafman, K. J. Holyoak, \& F. Boller (Eds.), Structure and functions of the human prefrontal cortex (Annals of the New York Academy of Sciences, Vol. 769, pp. 253-263). New York: New York Academy of Sciences.

JoHnson, M. K. (1991). Reality monitoring:Evidence from confabulation in organic brain disease patients. In G. P. Prigatano \& D. L. Schacter (Eds.), Awareness of deficit after brain injury: Clinical and theoretical issues (pp. 176-197). New York: Oxford University Press.

Johnson, M. K., Hashtroudi, S., \& LindSAY, S. (1993). Source monitoring. Psychological Bulletin, 114, 3-28.

Jones, T. C., JACOBY,L. L. \& GelLis, L. A. (2001). Cross-modal feature and conjunction errors in recognition memory. Journal of Memory \& Language, 44, 131-152.

Loftus, E. F., Miller, D. G., \& Burns, H. J. (1978). Semantic integration of verbal information into a visual memory. Journal of Experimental Psychology: Human Learning \& Memory, 4, 19-31.

Loftus, G. R, \& Masson, M. E. J. (1994). Using confidence intervals in within-subject designs. Psychonomic Bulletin \& Review, 1, 476-490.

McDermott, K. B. (1996). The persistence of false memories in list recall. Journal of Memory \& Language, 35, 212-230.

Moscovitch, M. (1995). Confabulation. In D. L. Schacter (Ed.), Memory distortion. Cambridge, MA: Harvard University Press.

Pezdek, K., \& Finger, K. (1997, November). The effect of verbal description on face recognition: "Release from verbal overshadowing." Paper presented at the 38th Annual Meeting of the Psychonomic Society, Philadelphia.

PINKER, S. (1997). How the mind works. New York: Norton. 
Reinitz, M. T., \& Hannigan, S. L. (2001). Effects of simultaneous stimulus presentation and attention switching on memory conjunction errors. Journal of Memory \& Language, 44, 206-219.

Reinitz, M. T., Lammers, W. J., \& Cochran, B. P. (1992). Memoryconjunction errors: Miscombination of stored stimulus features can produce illusions of memory. Memory \& Cognition, 20, 1-11.

Ross, D. F., Ceci, S. J., Dunning, D., \& Toglia, M. P. (1994). Unconscious transference and mistaken identity: When a witness misidentifies a familiar but innocent person. Journal of Applied Psychology, 79, 918-930.

Shank, R. C., \& Abelson, R. P. (1977). Scripts, plans, goals, and understanding. Hillsdale, NJ: Erlbaum.
Spaniol, J., \& Bayen, U. J. (2002). When is schematic knowledge used in source monitoring? Journal of Experimental Psychology: Learning, Memory, \& Cognition, 28, 631-651.

Tulving, E. (1985). Memory and consciousness. Canadian Psychology, 26, 1-12.

Wharton, C. M., Holyoak, K. J., \& Lange, T. E. (1996). Remote analogical reminding. Memory \& Cognition, 24, 629-643.

(Manuscript received August 12, 2002;

revision accepted for publication January 28, 2003.) 